\title{
Impact of Caregiving on Various Aspects of the Lives of Caregivers
}

\author{
Babar Irfan ${ }^{1}$, Omar Irfan ${ }^{2}$, Ahmed Ansari ${ }^{3}$, Waris Qidwai ${ }^{4}$, Kashmira Nanji ${ }^{5}$ \\ 1. Medicine, Jinnah Sindh Medical University (SMC) 2. Medicine, The Aga Khan University 3. Medical \\ College, The Aga Khan University, Karachi, PAK 4. Family Medicine, The Aga Khan University 5. \\ Epidemiology and Public Health, The Aga Khan University, Karachi, PAK
}

$\square$ Corresponding author: Kashmira Nanji, kashmira.nanji@gmail.com Disclosures can be found in Additional Information at the end of the article

\section{Abstract \\ Objective}

This study was designed to assess the impact of caregiving on the lives of the caregivers.

\section{Methods}

A cross-sectional study was conducted between July and September 2015 at a teaching hospital in Karachi, Pakistan. Participants who were more than 18 years old and were involved in caregiving (former or current) of a family member were invited to participate in the study. The participants were recruited through consecutive sampling technique. A total of 400 caregivers were interviewed. Written informed consent was obtained from all the participants. A pretest structured questionnaire was used for data collection and included sections on demographic details and impact of caregiving on various aspects of the lives of caregivers. The data was analyzed using SPSS version 19 (IBM, NY, USA).

\section{Results}

Information about a total of 400 caregivers (215 men and 185 women) was included in the final analysis. The majority (57.0\%) of the participants were aged between 18 and 30 years. About three-fifths (60\%) of the participants were single and the majority of the participants were students. Approximately $64 \%$ of the participants were currently involved in caregiving and about $48 \%$ of the participants responded that caregiving has an overall negative impact on various aspects, such as physical (40.8\%), psychological (47.8\%), and professional aspects (51.8\%) of their lives.

\section{Conclusion}

Received 04/17/2017

Review began 04/20/2017 Review ended 04/26/2017 Published 05/02/2017

C Copyright 2017

Irfan et al. This is an open access article distributed under the terms of the Creative Commons Attribution License CC-BY 3.0., which permits unrestricted use, distribution, and reproduction in any medium, provided the original author and source are credited.
Negative impact of caregiving was observed among caregivers due to extensive demands of caregiving and limited resources. Therefore, it is imperative for health care providers to explore, identify and support caregivers to cope in a better way to the challenging task of caregiving.

Categories: Family/General Practice, Public Health, Epidemiology/Public Health Keywords: caregiving, caregiver, impact, health, pakistan

Introduction 
A caregiver is someone who provides care to a person who is incapacitated or handicapped [1]. In general, care can be given to anyone; it can be a spouse who has suffered from a myocardial infarction; a parent with Alzheimer's disease; a mother-in-law with a malignancy, or a child with traumatic injury caused during sports. Informal caregivers include family members and friends who provide care to their dear ones without any financial benefits; whereas, formal caregivers are volunteers or paid care providers associated with a service system [1].

Women constitute more than half ( $59 \%$ to $75 \%$ ) of the caregiver population [2]. Evidence from studies suggests that women caregivers are usually involved in physically demanding tasks (i.e. bathing, toileting, and dressing) as compared to their male counterparts, who are more likely to provide financial support [3].

During the period of caregiving, the caregivers experience stress and burden resulting from the rigorous activity of caregiving, which can have a negative impact on their physical, psychological, and social lives, thereby decreasing their quality of life (QOL) [4]. Thus, caregivers are at risk of developing psychiatric disorders [5]. Caregiver burden has been proven to account for poor physical and emotional health [5]. Studies on caregivers suggest that caregiving is associated with psychological complaints such as depression and poor physical and psychological QOL [6-8]. A recent study concluded that caregiving was associated with distress, anxiety, stress, and depression [9].

Caregiving also has positive outcomes such as appreciation from patients, improved family cohesion, developing resilience, and gaining a sense of self-worth and accomplishment [10]. These positive aspects of caregiving have been found to be associated with lower levels of caregiver burden for life [11].

There is limited data regarding impact of caregiving on the lives of the caregivers in Pakistan. Therefore, this study was designed to identify the impact of caregiving on various aspects of the lives of the caregivers.

\section{Materials And Methods}

A cross-sectional study was conducted between July and September 2015 at a teaching hospital in Karachi, Pakistan. A total of 400 participants who were 18 years or older and were currently or formerly involved in caregiving to a family member were recruited through consecutive sampling technique. Written informed consent was obtained from the study participants after explaining the study protocol to them. The study protocol was reviewed and approved by the Departmental Research Committee of the Aga Khan University.

A pretested structured questionnaire was used as the data collection tool. The questionnaire was formed after thorough literature search and was reviewed by the authors. It was administered in both English and Urdu languages. The questionnaire was composed of two sections. The first section included demographic details of the participants such as age, gender, educational status, occupation, current caregiver, time since caregiving, and relation with the care recipient. Section 2 of the questionnaire included impact of caregiving on different aspects (physical, psychological, family, professional, and social) of the lives of the caregivers. The data was entered and analyzed using SPSS version 19.0 (IBM, NY, USA). Frequencies were obtained for all variables of interest. The results were presented in the form of frequencies and percentages.

\section{Results}

Information from a total of 400 participants was included in the final analysis. Table 1 presents 


\section{Cureus}

the sociodemographic characteristics of the study participants. About $54 \%$ of the participants were male and $46 \%$ were female. Amongst the participants, the majority (57\%) were between 18 and 30 years of age. Slightly under $59 \%$ of the participants were single. Sixty-four percent of the participants responded that they were currently involved in caregiving while $36 \%$ of the participants responded that they were involved in caregiving in the past five years. Most of the caregivers informed that they were giving care to their parents or grandparents; $39 \%$ were giving care to their mother and $26 \%$ were giving care to their father.

\section{Variables}

Frequency

\section{Percentage}

Age

$18-30$

Gender

Marital status

Single

Care status

Current

Duration of Care

Occupational Status

Educational Status 


\section{Cureus}

Matric \& intermediate

Graduate

Post Graduate
180

116

85
45.0

29.0

21.2

\section{TABLE 1: Sociodemographic Characteristics of Study Participants $(n=400)$}

Table 2 depicts the impact of caregiving on the life of caregivers. About $46 \%$ of the participants responded that caregiving had adversely impacted their life. The daily routine, such as sleep, eating, exercise, of the majority of the participants (65\%) were affected by caregiving. In addition, caregiving had an adverse impact on the physical (40.8\%), psychological (47.8\%), family (48.5\%), work (51.3\%) and social (53\%) life of the participants.

\section{Questions/Response}

Caregiving adversely impact life of caregiver?

Yes

No

Not sure

Caregiving adversely impact physical life of caregiver?

Yes

No

Not sure

Caregiving adversely impact psychological life of caregiver?

Yes

No

Not sure

Caregiving adversely impact family life of caregiver?

Yes

No

Not sure

Caregiving impacts professional/work life of caregiver?

Yes

143

$\%$

No 


\section{Cureus}

Not sure

Caregiving adversely impact social life of caregiver?

Yes

No

Not sure

Caregiving adversely impact financial status of caregiver?

Yes

No

Not sure

Caregiving adversely impact daily routine of caregiver such as sleep, eating, exercise?

Yes

No

Not sure

Caregiving adversely impact recreational life of caregiver?

Yes

Caregiver often neglects his/her own health during caregiving?

Yes

TABLE 2: Responses of the Participants on the Impact of Caregiving on the Life of Caregivers $(\mathrm{N}=400)$

\section{Discussion}

Caregiving includes assistance with activities of daily living, helping in medical care (e.g. medication management and accompanying patients to the hospital), and providing emotional and financial support [12]. In our study, the majority of the caregivers were males, that is, they were mainly providing the financial support. This is consistent with our tradition and culture where males provide financial support to the family as mostly they are the sole bread earners. Moreover, the majority of the caregivers were students enrolled in universities for graduation or post-graduation degrees. The potential negative effects on caregivers can extend well beyond mental and physical health. A study demonstrated that caregivers, particularly younger caregivers, often neglect their education, putting education on hold or dropping out 
entirely, which can impact their future career [13].

Almost half of the participants in the study responded that they suffered from financial difficulties while providing care; similar responses were recorded from a survey which concluded that caregiving results in considerable financial strain, and can cause difficulties in fulfilling other responsibilities such as marriage and employment [14]. In the current study, about $51.3 \%$ individuals reported that their professional work life has been negatively affected due to caregiving. It is believed that a good income and a high socioeconomic status can have a decreased impact on the caregivers' financial constraints.

Studies have shown that illness of a close relative causes distress and compromises the caregiver's health to a greater extent [15]. We report that $61.3 \%$ individuals in the study neglected their health during caregiving. This is consistent with the results of previous studies which had concluded that caregivers may be particularly vulnerable because of caregiving demands that can lead to compromising their physical health and can exacerbate existing chronic health conditions [16]. Dementia caregivers report more depression than any other caregivers [17]. Due to lack of time, the caregivers are less likely to care for themselves and may be prone to certain medical conditions [18].

Our study results show that caregiving has an overall impact on daily life as $65 \%$ of the participants reported to have altered sleeping and eating habits. A meta-analysis of the physical and mental health effects of caregiving have concluded that higher levels of depression and physical health problems were observed among caregivers as compared with non-caregivers [19].

Some studies have shown contrasting results emphasizng the positive effects of caregiving. These studies report that caregivers may even experience improved health status because of their caregiving experience. However, there are clearly subgroups of caregivers who report high levels of stress and experience depression from caregiving.

Caregiving can be a very challenging task where caregivers do not have enough resources such as knowledge, skills, social support and face a lack of respite and community services.

The study had several potential limitations. Since this was a cross-sectional study, causality was difficult to establish. Moreover, in this study we did not focus on a specific disease or ailment, as the caregiver burden and impact might get changed due to different diseases. The data was collected from an urban city of Pakistan; therefore, the results may vary when compared to the data from people living in rural areas.

\section{Conclusions}

In conclusion, our study shows the negative impact of caregiving on various aspects of the lives of the caregiver. This could provide an evidence basis for the development of support programs designed for caregivers. Health care professionals should be prepared to address the caregivers' psychological, social, and health needs. They should also highlight the positive aspects of caregiving such as a feeling of pride, a sense of purpose, and satisfaction received from giving care to dear ones.

\section{Additional Information \\ Disclosures}

Human subjects: Consent was obtained by all participants in this study. Animal subjects: All authors have confirmed that this study did not involve animal subjects or tissue. Conflicts of 
interest: In compliance with the ICMJE uniform disclosure form, all authors declare the following: Payment/services info: All authors have declared that no financial support was received from any organization for the submitted work. Financial relationships: All authors have declared that they have no financial relationships at present or within the previous three years with any organizations that might have an interest in the submitted work. Other relationships: All authors have declared that there are no other relationships or activities that could appear to have influenced the submitted work.

\section{Acknowledgements}

We are thankful to all the participants and administrative staff of the clinics.

\section{References}

1. Gouin JP, da Estrela C, Desmarais K, et al.: The impact of formal and informal support on health in the context of caregiving stress. Fam Relat. 2016, 65:191-206. 10.1111/fare.12183

2. Paraponaris A, Davin B, Verger P: Formal and informal care for disabled elderly living in the community: an appraisal of French care composition and costs. Eur J Health Econ. 2012, 13:327-336. 10.1007/s10198-011-0305-3

3. DePasquale N, Davis KD, Zarit SH, et al.: Combining formal and informal caregiving roles: the psychosocial implications of double-and triple-duty care. J Gerontol B Psychol Sci Soc Sci. 2016, 71:201-211. 10.1093/geronb/gbu139

4. Caputo J, Pavalko EK, Hardy M: The long term effects of caregiving on women's health and mortality. J Marriage Fam. 2016, 78:1382-1398. 10.1111/jomf.12332

5. Mosquera I, Vergara I, Larranaga I, et al.: Measuring the impact of informal elderly caregiving: a systematic review of tools. Qual Life Res. 2016, 25:1059. 10.1007/s11136-015$1159-4$

6. Prince M, Brodaty H, Uwakwe R, et al.: Strain and its correlates among carers of people with dementia in low-income and middle-income countries. A 10/66 Dementia Research Group population-based survey. Int J Geriatr Psychiatry. 2012, 27:670-682.

7. Hansen T, Slagsvold B: Feeling the squeeze? The effects of combining work and informal caregiving on psychological well-being. Eur J Ageing. 2015, 12:51-60. 10.1007/s10433-0140315-y

8. Vitaliano PP, Strachan E, Dansie E, et al.: Does caregiving cause psychological distress? The case for familial and genetic vulnerabilities in female twins. Ann Behav Med. 2014, 47:198207. 10.1007/s12160-013-9538-y

9. Gupta S, Isherwood G, Jones K, et al.: Assessing health status in informal schizophrenia caregivers compared with health status in non-caregivers and caregivers of other conditions. BMC Psychiatry. 2015, 15:162. 10.1186/s12888-015-0547-1

10. Bauer R, Sterzinger L, Koepke F: Rewards of caregiving and coping strategies of caregivers of patients with mental illness. Psychiatr Serv. 2013, 64:185-188. 10.1176/appi.ps.001212012

11. Pinquart M, Sorensen S: Associations of stressors and uplifts of caregiving with caregiver burden and depressive mood: a meta-analysis. J Gerontol B Psychol Sci Soc Sci. 2003, 58:11228.

12. Adelman RD, Tmanova LL, Delgado D, et al.: Caregiver burden: a clinical review . JAMA. 2014, 311:1052-1060. 10.1001/jama.2014.304

13. Adler R, Mehta R: Catalyzing technology to support family caregiving . National Alliance for Caregiving. 2014. 1-18.

14. Grady PA, Rosenbaum L: The science of caregiver health. J Nurs Scholarsh. 2015, 47:197-199. 10.1111/jnu.12137

15. Deshields TL, Rihanek A, Potter P, et al.: Psychosocial aspects of caregiving: perceptions of cancer patients and family caregivers. Support Care Cancer. 2012, 20:349-356.

10.1007/s00520-011-1092-1

16. Bakas T, Burgener SC: Predictors of emotional distress, general health, and caregiving outcomes in family caregivers of stroke survivors. Top Stroke Rehabil. 2015, 9:34-45. 10.1310/GNOJ-EXVX-KXOB-8X43

17. Roth DL, Fredman L, Haley W: Informal caregiving and its impact on health: a reappraisal 


\section{Cureus}

from population-based studies. Gerontologist. 2015, 55:309-19. 10.1093/geront/gnu177

18. Park B, Kim SY, Shin JY, et al.: Prevalence and predictors of anxiety and depression among family caregivers of cancer patients: a nationwide survey of patient-family caregiver dyads in Korea. Supportive Care Cancer. 2013, 21:2799-2807. 10.1007/s00520-013-1852-1

19. Pinquart M, Sorensen S: Differences between caregivers and non-caregivers in psychological health and physical health: a meta-analysis. Psychology. 2003, 18:250-267. 10.1037/08827974.18.2.250 